\title{
Single scattering detection in turbid media using single-phase structured illumination filtering
}

\author{
E. Berrocal \\ edouard.berrocal@forbrf.lth.se
}

\section{J. Johnsson}

E. Kristensson

M. Aldén
Department of Physics, Division of Combustion Physics, Lund University, Sweden

Department of Physics, Division of Combustion Physics, Lund University, Sweden

Department of Physics, Division of Combustion Physics, Lund University, Sweden

Department of Physics, Division of Combustion Physics, Lund University, Sweden

This work shows a unique possibility of visualizing the exponential intensity decay due to light extinction, when laser radiation propagates through a homogeneous scattering medium. This observation implies that the extracted intensity mostly originates from single scattering events. The filtering of this single light scattering intensity is performed by means of a single-phase structured illumination filtering approach. Results from numerical Monte Carlo simulation confirm the experimental findings for an extinction coefficient of $\mu_{e}=0.36 \mathrm{~mm}^{-1}$. This article demonstrates an original and reliable way of measuring the extinction coefficient of particulate turbid media based on sidescattering imaging. Such an approach has capabilities to replace the commonly used transmission measurement within the intermediate single-to-multiple scattering regime where the optical depth ranges between $1<O D<10$. The originality of the presented approach is that only one image is used (instead of three images usually employed in structured illumination) and that no monitoring of the incident intensity is required, simplifying the experimental procedure and set-up. Applications of the technique has potential in probing challenging homogeneous scattering media, such as biomedical tissues, turbid emulsions, etc, in situations where dilution cannot be applied and where conventional transmission measurements fail.

[DOI: http://dx.doi.org/10.2971/jeos.2012.12015]

Keywords: structured illumination, Monte Carlo simulation, multiple scattering, turbid media

\section{INTRODUCTION}

Elastic and inelastic scattering of laser radiation in turbid media is widely used for a variety of applications spanning from Biomedical Optics (treatment of cells by photodynamic, imaging of tissue phantoms, measurement of glucose and oxygen in blood [1], etc) to Combustion Engineering (study of twophase flows, characterization of spray systems [2], etc). Other examples of turbid media includes clouds, fog, smoke, suspensions, emulsions, colloidal solutions etc. One fundamental property, which describes the turbidity of a medium at a given wavelength, is the extinction coefficient $\mu_{e}$. Optical measurements of $\mu_{e}$ are usually performed by means of either transmission [3] or back-scattering measurements [4]. In the case of back-scattering detection (e.g. used in Lidar measurements) the desired signal arises from singly scattered photons only, while in the case of transmission detection (e. g. used for extinction measurements) the desired signal arises from ballistic photons only. These line-of-sight configurations are attractive as they can directly take advantage of the light properties of the incident beam (such as polarization, direction of propagation, wavelength, pulse duration or coherence length) in order to extract the desired signal from the multiple light scattering. However, for the side-scattering detection, where time-gating and time-resolved schemes are hardly applicable and where the suppression of the multiply scattered photons is challeng- ing, quantitative measurement of $\mu_{e}$ has been usually avoided. Note, nevertheless, that Monte Carlo calculations have been performed in the past [5] to estimate and optimize the amount of single light scattering in respect to multiple light scattering for various crossed source-detector geometries. Even though these numerical results had provided valuable further knowledge in optimizing the detection of singly scattered photons, no experimental solution was provided to filter out the detected multiple light scattering intensity.

Since 2008, a novel side-scattering approach called Structured Laser Illumination Planar Imaging (SLIPI) has been developed for removing light intensity introduced by multiply scattered photons, leading to significant image contrast enhancement $[6,7]$. This filtering approach has also been used in microscopy for the imaging of an adult fruit fly [8] and for the early development of a zebrafish [9]. In 2010, the possibility of using the technique to freeze fast events (within few hundreds of nanosecond), has been demonstrated in the case of a transient hollow-cone spray [10]. More recently the technique has been tested in various homogeneous scattering and fluorescing media [11], as well as for two-dimensional extraction of the extinction coefficient in an inhomogeneous spray system [12]. The method is based on using an incident laser sheet 
with a sinusoidal intensity pattern along the vertical direction. While the multiply scattered light is mostly independent of the modulation pattern, the singly scattered light preserve it faithfully. This implies that the amplitude of the modulated component is a direct signature of the single light scattering. The SLIPI process consists, then, in extracting this amplitude by recording several structured images where a spatial phase shift is induced by vertically displacing the modulated pattern between each image. In the previous references [6]-[12], the resultant SLIPI image was reconstructed by means of three modulated images. Nevertheless, the technique can be generalized to a larger number $n$ of modulated images. By vertically shifting the incident modulation $(n-1)$ times, a series of $n$ modulated images $I$ are recorded with a spatial phase shift of the modulation of $\triangle \Phi=2 \pi / n$ between each successive image. Based on these $n$ modulated images, the resulting SLIPI image $S$ is constructed according to:

$$
S=\frac{\sqrt{2}}{n}\left(\sum_{j=1}^{n-1} \sum_{k=j+1}^{n}\left(I_{j}-I_{k}\right)^{2}\right)^{1 / 2}
$$

where $I_{j}$ and $I_{k}$ are intensity values from the modulated images and the subscripts $j$ and $k$ denote the different image recordings for different phases of the modulation. Here, the pair-wise subtraction $\left(I_{j}-I_{k}\right)$ removes similar features introduced by the multiple light scattering while unique features from single light scattering are kept. Note that this equation extracts the amplitude of the modulation while preserving the spatial resolution, only if $n \geq 3$. This condition of requiring a minimum of 3 phases is due to the sinusoidal spatial pattern of the incident illumination.

In this article an original approach to extract the amplitude of the modulation $S$ from only one phase/image is presented. The technique is tested both experimentally and by means of numerical Monte Carlo simulation. First, the experimental measurement is detailed. Second, a description of the simulation is given. Finally, experimental and simulated results are provided and compared.

\section{EXPERIMENTAL SET-UP}

The experimental set-up, shown in Figure 1, consists in illuminating a homogeneous scattering medium using a spatially modulated laser sheet and imaging it at $90^{\circ}$. The probed volume is a cuvette containing a homogeneous distribution of quasi-monodisperse and non-absorbing polystyrene spheres (2.66\% solids-suspension from Polysciences Inc.) immersed in distilled water. The diameter of the scattering spheres is $0.486 \mu \mathrm{m}$ with $0.011 \mu \mathrm{m}$ standard deviation. The investigated solution was created by diluting the solids-suspension of $4.12 \cdot 10^{11}$ particles $/ \mathrm{ml}$ in concentration, with distilled water.

The incident light is produced by a pulsed Nd:YAG laser emitting $\lambda=532 \mathrm{~nm}$ radiation at $10 \mathrm{~Hz}$. Pulses of $10 \mathrm{~ns}$ are used at $30 \mathrm{~mJ}$ energy to illuminate the sample. The modulated laser sheet is constructed by means of a $10 \mathrm{lp} / \mathrm{mm}$ Ronchi grating and using a succession of cylindrical lenses as previously de-

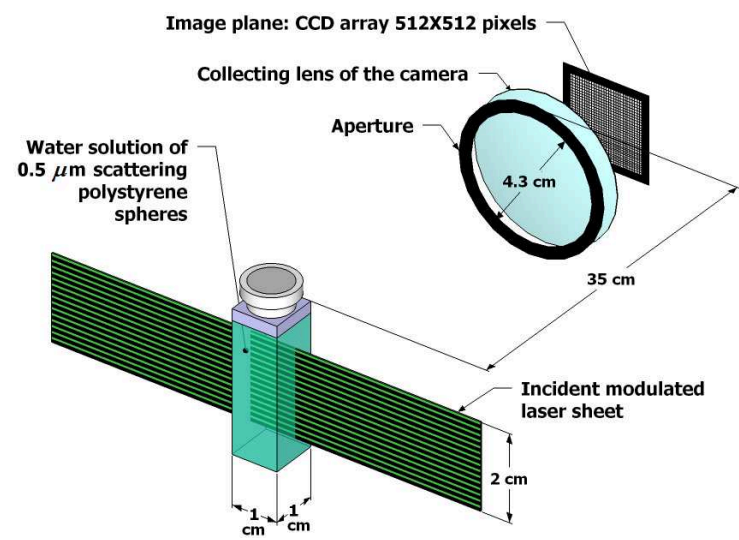

FIG. 1 Description of the experimental setup: A laser sheet, spatially modulated in intensity, crosses a homogeneous scattering solution containing $0.486 \mu \mathrm{m}$ polystyrene spheres. The laser sheet is imaged at $90^{\circ}$ using an EM-CCD camera.

scribed in $[6,10]$. However, contrary to the optical arrangement shown in $[6,10]$, no tilted glass plate is used in the present work, as a shift of the modulation is not required here. The resulting spatial period of the modulation equals $500 \mu \mathrm{m}$ and the height of the laser sheet is $2.0 \mathrm{~cm}$. The light sheet is positioned at a distance $d=2$ or $d=4 \mathrm{~mm}$ from the cuvette side. These configurations allow the efficiency of the filtering process to be investigated for various amounts of multiple light scattering detected.

The light intensity scattered from one side of the cuvette is detected using an Andor iXon DV887 Electron Multiplying CCD camera. This camera has single photon detection capability without an image intensifier due to its multiplication gain feature. At $532 \mathrm{~nm}$, the quantum efficiency reaches $90 \%$ when using the thermoelectric cooling set to $-20^{\circ} \mathrm{C}$. A $1.0 \mathrm{~cm} \times 1.0 \mathrm{~cm}$ surface, corresponding to the central region of the laser sheet, is imaged onto $228 \times 228$ pixels, resulting in an image resolution of $44 \mu \mathrm{m} /$ pixel. To increase the signal to noise ratio 500 single-shots images are averaged to create the final modulated image. In front of the $5.0 \mathrm{~cm}$ diameter collecting lens, an aperture is used to fix the surface of detection corresponding, here, to a disc of $4.3 \mathrm{~cm}$ in diameter. The distance, $L$, between the surface of the cell and the aperture equals $35 \mathrm{~cm}$. From these parameters, the detection acceptance angle of the collection optics equals $\theta_{a}=5.2^{\circ}$.

In this experiment, an extinction coefficient of $0.36 \mathrm{~mm}^{-1}$ was experimentally measured using a single-phase structured illumination filtering approach. The goal of this work is, then, to verify if similar results can be obtained from numerical Monte Carlo simulation using a model already validated (see reference [2]). This is performed here by simulating respectively:

- The propagation of the modulated laser sheet through the scattering cell

- The detection of the scattered light by the collecting lens

- The image formation on a pixel array

- The measurement of the extinction coefficient from the simulated image 
The comparison between the experimental and simulated results, aims to provide indications regarding the efficiency of the presented single-phase structured illumination filtering process, for the extraction the single light scattering intensity only.

\section{MONTE CARLO SIMULATION}

The propagation of light radiation through the scattering volume is simulated using the statistical Monte Carlo (MC) method. The implementation of MC simulation for photon transport through turbid media has been treated in the past by a large body of literature, especially within the field of Biomedical Optics [13, 14]. The successive calculation steps as well as a detailed description and validation of the $\mathrm{MC}$ code used in this work can be found in $[2,15]$. In order to match the experimental set-up described in the subsection above, the dimensions of the simulated scattering medium are $1.0 \mathrm{~cm} \times 1.0 \mathrm{~cm} \times 2.0 \mathrm{~cm}$ as illustrated in Figure 2. The variation of the incident light intensity is simulated by means of photon weighting factors stored in an input matrix. A sinusoidal pattern is given along the vertical direction $\mathrm{Z}$, while a Gaussian intensity distribution is applied along the $X$ direction. The resultant modulated laser sheet is $2.0 \mathrm{~cm}$ high, with

(a)

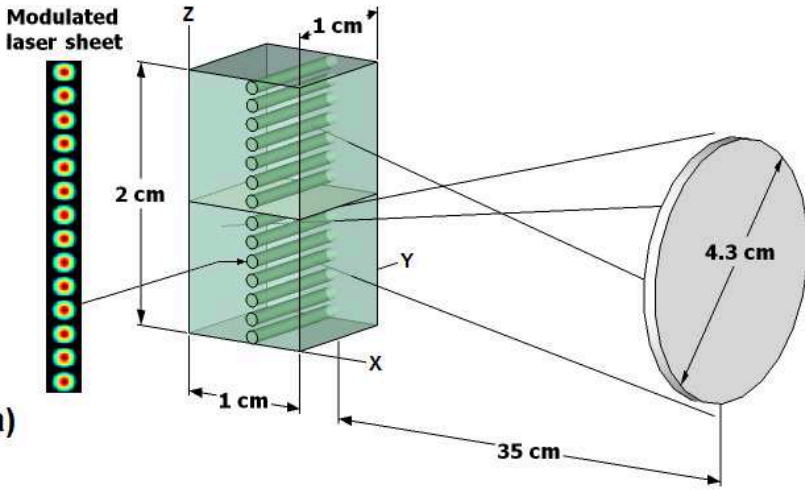

(b)

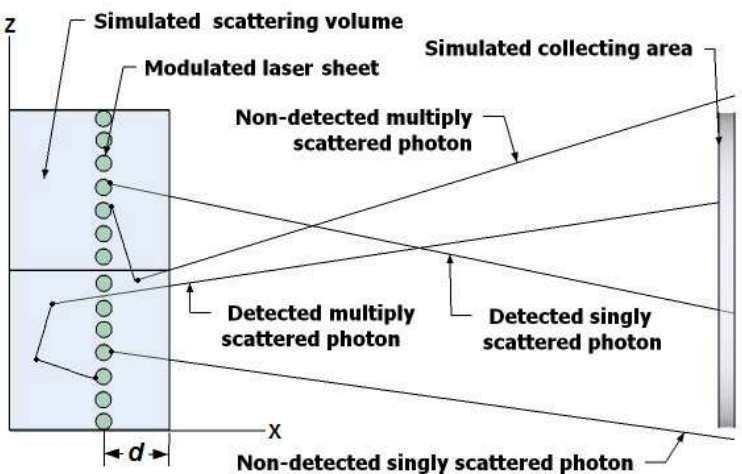

FIG. 2 Description of the numerical simulation: (a) Two homogeneous cubic volumes are used to reconstruct a $1.0 \mathrm{~cm} \times 1.0 \mathrm{~cm} \times 2.0 \mathrm{~cm}$ scattering volume. The modulated incident laser sheet is simulated by means of an input matrix. Photon transport through the scattering volume is modeled by means of MC simulation. The side view of illustration (a) is shown in (b), highlighting different photon paths prior to detection. Photons reaching the collecting area are selected to form a one-to-one image of the laser sheet plane using a ray-tracing approach. The simulated image is formed on a plane (not shown on the figure) where the spatial resolution of the matrix matches the one of the experiment. a full width at half maximum of $500 \mu \mathrm{m}$. The refractive index of the scattering particles is determined from the experimental results published by Ma et al. [16]. According to the authors the refractive index of polystyrene spheres illuminated at $532 \mathrm{~nm}$, is $n_{p}=1.588-0.0003 i$. In the simulation a real (non-absorbing) index of refraction is assumed for the scatterers, such that $n_{p}=1.59-0.0 i$.

The same assumption applies for the surrounding medium composed of distilled water, where $n_{w}=1.33-0.0 i$. The scattering particles were assumed perfectly spherical and monodispersed with a diameter of $0.5 \mu \mathrm{m}$. The resulting scattering phase function of a single polystyrene sphere is computed based on the Lorenz-Mie theory $[17,18]$ and is shown in Figure 3. The cumulative density function of the scattering phase function is used in the MC code to randomly define the new photon direction of propagation after each scattering event. All photons exiting the detection side face of the scattering volume are further traced by means of a ray-tracing approach. Photons reaching a $4.3 \mathrm{~cm}$ diameter disk located at 35 $\mathrm{cm}$ from the scattering volume (simulating the experimental collecting lens) are selected and further traced to form a oneto-one image. The matrix size of the simulated image is fixed to match the experimental image resolution of $44 \mu \mathrm{m} /$ pixel. Note that the use of the ray-tracing approach is required to reproduce and visualize the modulation pattern of the incident laser sheet. In order to verify the experimental measurement, the scattering coefficient is set to $\mu_{e}=0.36 \mathrm{~mm}^{-1}$ resulting in an optical depth of 3.6 over the full path through the cuvette. The laser sheet is located at distance $d=2 \mathrm{~mm}$ from the detection face of the cube and a total of 75 billion photons are launched in the simulation.

\section{RESULTS}

The experimental results are presented in Figure 4. In (a) the distance between the laser sheet and the detection side of the cuvette is $d=2 \mathrm{~mm}$, while in (b) $d=4 \mathrm{~mm}$. Due to attenua-

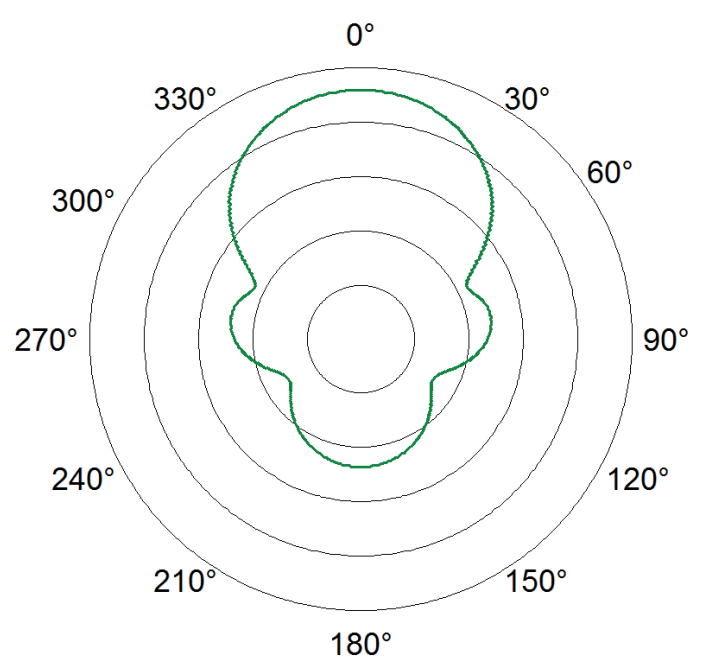

FIC. 3 Polar scattering phase function (logarithmic scale) computed based on the Lorenz-Mie theory $[16,17]$ for a $0.5 \mu \mathrm{m}$ polystyrene sphere immersed in pure water and illuminated at $532 \mathrm{~nm}$. The refractive indices of the sphere and of the water are respectively equal to $n_{p}=1.59-0.0 i$ and $n_{w}=1.33-0.0 i$. 
(a)
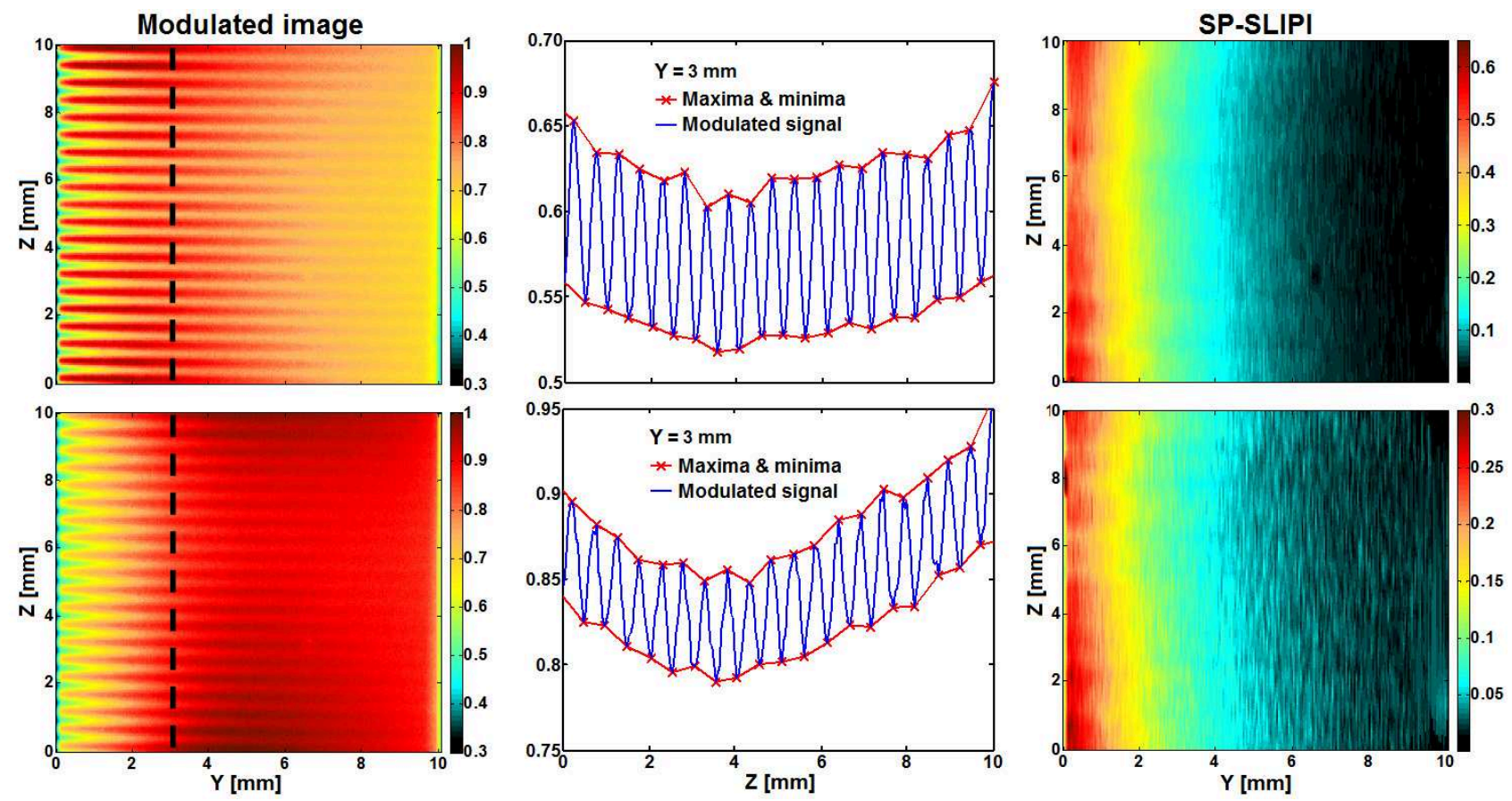

FIG. 4 Experimental results: In (a) the distance between the laser sheet and the detection side of the cuvette is $d=2 \mathrm{~mm}$, while in (b) $d$ is increased to 4 mm, corresponding respectively to an optical depth of $O D=0.72$ and $O D=1.44$ between the laser sheet and the exit of the scattering cell. On the left side, images of the modulated laser sheet crossing the scattering cuvette are shown. In the middle of the figure, the light intensity detected along a single pixel column, at $y=3$ mm (along the black dashed line), is plotted. The modulated component represents the single light scattering intensity while the intensity offset is the contribution of the multiple light scattering intensity. Interpolations in between maximum and in between minimum values are performed by means of a peak detection algorithm. Based on these interpolation curves, the amplitude of the modulation can be extracted for each pixel column (between 0 and $10 \mathrm{~mm}$ ) resulting in the SP-SLIPI images given on the right side.

tion effects, it is seen from the central plots that the amplitude of the modulation at a given distance $y$ (e.g. at $y=3 \mathrm{~mm}$, along the black dashed line) is lower for $d=4 \mathrm{~mm}$ than for $d=2 \mathrm{~mm}$. This attenuation corresponds to an optical depth of $O D=1.44$ and $O D=0.72$ respectively.

Based on a single modulated image (see left images in Figure 4), the goal of Single-Phase Structured Laser Illumination Planar Imaging (SP-SLIPI) is to estimate, the amplitude of the modulation along each pixel column. This is performed, here, by first localizing the positions of the maxima and the minima for a given column. This localization process is made by Fourier transforming the pixel column and, guided by the known spatial frequency of the modulation, finding and extracting the frequency and phase of the modulation within the column. The frequency and phase of the modulation are employed to localize the positions of the maxima and minima where a linear interpolation is used to connect them (see central plots in Figure 4), respectively. The lines connecting the minima can then be subtracted from the lines connecting the maxima, resulting in an estimate of the amplitude as a function of the position in the pixel column. It should be noted that the spatial resolution of the estimated amplitude, over the pixel column, is reduced compared to the initial resolution of the modulated image. This loss in image resolution is dependent on the spatial frequency of the modulation (higher spatial frequency results in lower resolution loss). The procedure results in the SP-SLIPI images given on the right side of Figure 4, where a light intensity decay from the entrance to the exit of the cell can be noticed. By comparing the experimental SP-SLIPI images at $d=2 \mathrm{~mm}$ in Figure 4(a) and $d=4 \mathrm{~mm}$ in Figure 4(b), it is observed that this light intensity decay is similar up to $y \sim 5 \mathrm{~mm}$. However, at larger distance $y$ the SP-SLIPI image becomes slightly more noisy in the case of $d=4 \mathrm{~mm}$ where the optical depth between the laser sheet and the camera is twice as high (from 0.72 to 1.44). This is explained by the fact that the remaining modulation is of the same order as the camera noise. Such effects limit the extraction of the amplitude of the modulation.

The results from the MC simulation are presented in Figure 5 where the distance between the laser sheet and the detection side is $d=2 \mathrm{~mm}$. In (a), all photons reaching the collecting lens are detected to form a one-to-one image of the modulated laser sheet on a matrix array, as shown on the left side of the figure. The central image shows the simulated SP-SLIPI calculated in the exact same way as in the experiment (see explanation above). On the right side of the figure a comparison between simulated and experimental results, for the SP-SLIPI intensity signal integrated along $Z$, is given. It is observed that both curves follow an exponential decay as predicted by the Beer-Lambert law, such as:

$$
I_{f}=I_{i} \cdot \exp \left(-\mu_{e} \cdot l\right)
$$

where $I_{f}$ and $I_{i}$ are the respective final and incident light intensity and $l$ is the distance along the axis of light propagation.

The observed exponential decay indicates, then, the efficient detection of the single light scattering intensity. An exponential fit reveals $\mu_{e}=0.32 \mathrm{~mm}^{-1}$ and $\mu_{e}=0.36 \mathrm{~mm}^{-1}$ for the 
(a)

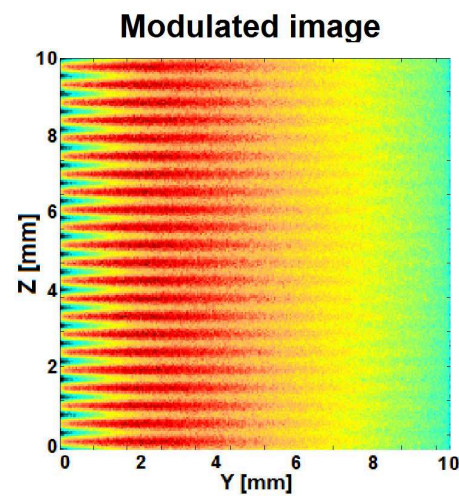

Single scattering
modulated image

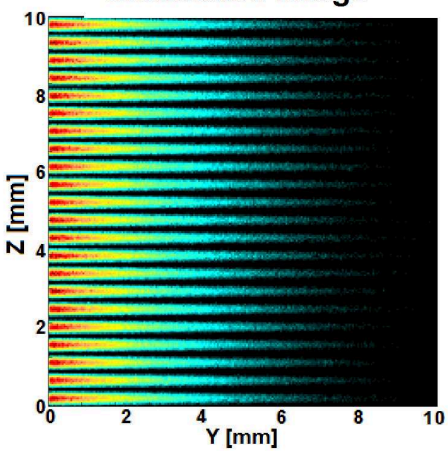

SP-SLIPI

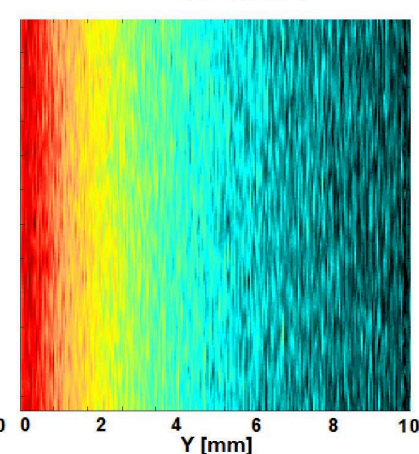

Single scatetring SP-SLIPI

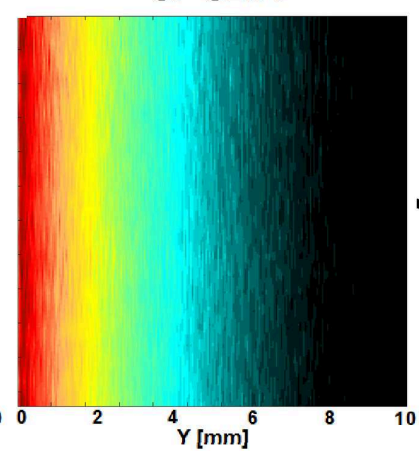

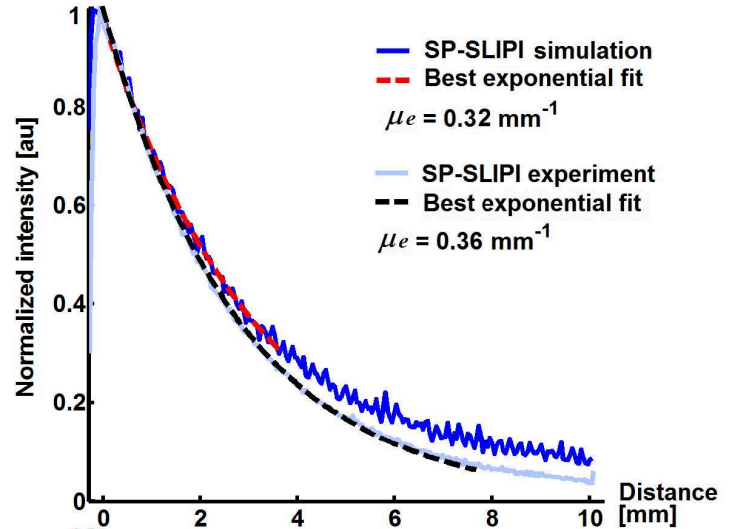

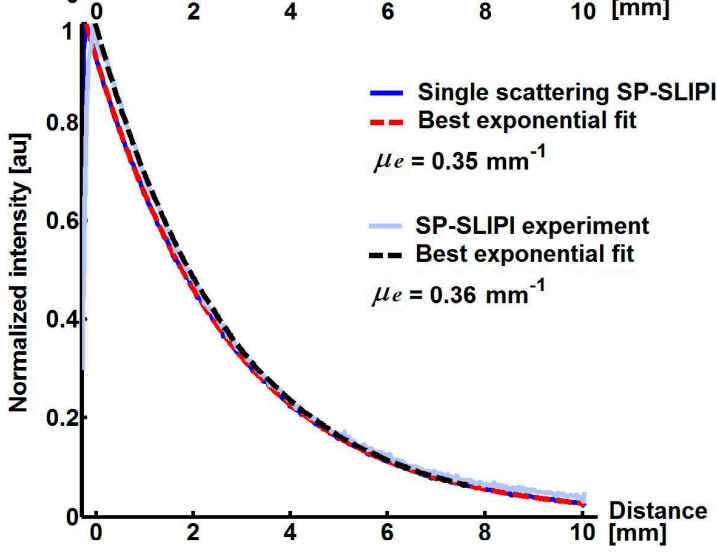

FIG. 5 Results from MC simulation: In (a) all photons are detected while in (b) the single light scattering intensity only is detected. The distance between the laser sheet and the detection side of the cuvette is $d=2 \mathrm{~mm}$ corresponding to an optical depth of $O D=0.72$ between the laser sheet and the exit of the scattering cell. The images in the center corresponds to the resultant SP-SLIPI images. The plots on the right side show the light intensity of the SP-SLIPI images integrated along the vertical direction. Each plot have been normalized to their own maxima value (as only the decay of the plots is of interest to extract $\mu_{e}$ and not the actual absolute light intensity value). A comparison between the simulated and experimental results are shown together with an estimate of the extinction coefficient extracted from the best exponential fit.

simulated and experimental curves respectively. A part of the divergence between these two values is related to the lack of photon statistics, making the simulated SP-SLIPI signal noisy, even though a large number of photons have been launched (75 billion). When, however, extracting the single light scattering intensity in the MC simulation, as shown in Figure 5(b), a non-noisy SP-SLIPI signal is obtained. This can be explained as the numerical suppression of the multiple light scattering is making the single light scattering always reaching minima of zero and only the maxima are affected by the statistical noise. As a result the extinction coefficient deduced from the exponential fit of the single light scattering equals $\mu_{e}=0.35 \mathrm{~mm}^{-1}$, which is close to the $0.36 \mathrm{~mm}^{-1}$ used as input data in the simulation. These results from numerical MC simulation demonstrate that the extinction coefficient of $0.36 \mathrm{~mm}^{-1}$, initially measured experimentally with SP-SLIPI, is probably a slight underestimation of the real extinction coefficient of the probed medium. Despite this underestimation, the presented study confirms the possibility of extracting a measure of the extinction coefficient within the intermediate single-to-multiple scattering regime, a regime where results are usually affected by errors introduced by multiple light scattering. Furthermore, it can be predicted that an extinction coefficient of 10 orders of magnitude higher could be measured along $1 \mathrm{~mm}$ side length. Such measurement could be of interest to characterize denser scattering media. It can be pointed out that accurate results in SP-SLIPI require the detection of a non-noisy sinusoidal pattern. This is achieved experimentally by recording images with a sufficiently long time of exposure or by averaging several single-shot images (as performed in this article). If the camera used is not sensitive enough to obtain an acceptable signal-to-noise ratio from single-shot based images, the possibility of imaging rapid inhomogeneous flows by means of SP-SLIPI would be restricted.

\section{CONCLUSION}

In this article, both experimental and simulated results demonstrate a unique possibility of imaging the exponential light extinction of a laser sheet crossing a scattering sample from the recording of a single image. This was achieved for a suspension of $0.5 \mu \mathrm{m}$ scattering polystyrene spheres in distilled water, at scattering coefficient $\mu_{e}=0.36 \mathrm{~mm}^{-1}$. The approach is based on the detection of the maximum and minimum values of the modulated signal and is applicable when probing homogeneous media or when high spatial resolution is not required. The presented filtering process is fairly simple and capable of efficiently extracting the single light scattering intensity. However, for particles much larger that the incident wavelength, the detection of this single light scattering can be expected to be more challenging, due to the highly forward peak of the scattering phase function (see article [11]). In this case, an underestimation of the extinction coefficient would be obtained. Further advanced MC studies of type presented in this article are necessary to understand 
the complex combined effect of the scattering phase function, the frequency of the modulation, the concentration of scattering particles and the detection acceptance angle of the collecting lens. From these preliminary results, it is nevertheless believed that SP-SLIPI measurement could be an alternative solution to the traditional transmission detection, for the characterization of homogeneous scattering media.

\section{ACKN OWLEDGEMENTS}

The authors wish to thank the Centre for Combustion Science and Technology through the Swedish Foundation for Strategic Research and the Swedish Energy Agency for financial support. The Swedish Research Council (Vetenskapsrådet) is acknowledged for supporting the project 2011-4272. Also the European Research Council advanced grant for the project DALDECS (Development and Application of Laser Diagnostic Techniques for Combustion Studies) is acknowledged.

\section{References}

[1] V. V. Tuchin, Handbook of optical biomedical diagnostics (SPIE Press, Bellingham, 2002).

[2] E. Berrocal, Multiple scattering of light in optical diagnostics of dense sprays and other complex turbid media, (Ph.D. Thesis, Cranfield University, England, 2006).

[3] L. Wang, X. Liang, P. Galland, P. P. Ho, and R. R. Alfano, "True scattering coefficients of turbid matter measured by early-time gating," Opt. Lett. 20, 913-915 (1995).

[4] A. Ansmann, M. Riebesell, and C. Weitkamp, "Measurement of atmospheric aerosol extinction profiles with a Raman lidar," Opt. Lett. 15, 746-748 (1990).

[5] E. Berrocal, D. Y. Churmakov, V. P. Romanov, M. C. Jermy, and I. V. Meglinski, "Crossed source-detector geometry for a novel spray diagnostic: Monte Carlo simulation and analytical results," Appl. Optics 44, 2519-2529 (2005).

[6] E. Berrocal, E. Kristensson, M. Richter, M. Linne, and M. Aldén, "Application of structured illumination for multiple scattering suppression in planar laser imaging of dense sprays," Opt. Express 16, 17870-17881 (2008).
[7] E. Kristensson, E. Berrocal, M. Richter, S.-G. Pettersson, and M. Aldén, "High-speed structured planar laser illumination for contrast improvement of two-phase flow images," Opt. Lett. 33, 2752-2754 (2008).

[8] T. Breuninger, K. Greger, and E. H. Stelzer, "Lateral modulation boosts image quality in single plane illumination fluorescence microscopy," Opt. Lett. 32, 1938-1940 (2007).

[9] P. J. Keller, A. D. Schmidt, A. Santella, K. Khairy, Z. Bao, J. Wittbrodt, and E. H. Stelzer, "Fast high-contrast imaging of animal development with scanned light sheet-based structured-illumination microscopy," Nat. Methods 7, 637-642 (2010).

[10] E. Kristensson, E. Berrocal, M. Richter, and M. Aldén, “Nanosecond structured laser illumination planar imaging for single-shot imaging of dense sprays," Atomization and Sprays 20, 337-343 (2010).

[11] E. Kristensson, L. Araneo, E. Berrocal, J. Manin, M. Richter, M. Aldén, and M. Linne, "Analysis of multiple scattering suppression using structured laser illumination planar imaging in scattering and fluorescing media," Opt. Express 19, 13647-13663 (2011).

[12] E. Kristensson, E. Berrocal and M. Aldén, "Extinction coefficient imaging of turbid media using dual structured laser illumination planar imaging," Opt. Lett. 36, 1656-1658 (2011).

[13] L. Wang, S. L. Jacques, L. Zheng, “MCML - Monte Carlo modeling of light transport in multi-layered tissues," Comput. Methods Programs Biomed. 47, 131-146 (1995).

[14] C. Macdonald and I. V. Meglinski, "Backscattering of circular polarized light from a disperse random medium influenced by optical clearing," Laser Phys. Lett. 8, 324-328 (2011).

[15] E. Berrocal, D. L. Sedarsky, M. E. Paciaroni, I. V. Meglinski, and M. A. Linne, "Laser light scattering in turbid media Part I: Experimental and simulated results for the spatial intensity distribution," 0 pt. Express 15, 10649-10665 (2007).

[16] X. Ma, J. Lu, S. Brocks, K. Jacob, P. Yang and X.-H. Xin, “Determination of complex refractive index of polystyrene microspheres from 370 to 1610 nm," Phys. Med. Biol. 48, 4165-4172 (2003).

[17] C. Bohren, and D. Huffman, Absorption and scattering of light by small particles (Wiley, New York, 1983).

[18] H. C. van de Hulst, Light scattering by small particles (Dover, New York, 1981). 